\title{
Prompt Photon Production as a probe of Gluon Sivers Function
}

\author{
Siddhesh Padval* \\ Department of Physics, University of Mumbai, Mumbai, India \\ E-mail: siddhesh.padvalephysics.mu.ac.in
}

\section{Rohini M. Godbole}

Centre for High Energy Physics, Indian Institute of Science, Bangalore, India.

E-mail: rohinieisc.ac.in

\section{Abhiram Kaushik}

Institute of Mathematical Sciences, Chennai, India

E-mail: abhiramkbeimsc.res.in

\section{Anuradha Misra}

Department of Physics, University of Mumbai, Mumbai, India

E-mail: misraephysics.mu.ac.in

\begin{abstract}
We explore the possibility of using transverse single spin asymmetries arising in prompt photon production, through scattering of unpolarized beams of protons/leptons off transversely polarized proton target, as probes of Gluon Sivers Function. We present estimates of asymmetries at both RHIC and EIC energies within the generalized parton model (GPM) and color gauge invariant generalized parton model (CGI-GPM).
\end{abstract}

Light Cone 2019 - QCD on the light cone: from hadrons to heavy ions - LC2019

16-20 September 2019

Ecole Polytechnique, Palaiseau, France

\footnotetext{
*Speaker.
} 


\section{Introduction and formalism}

In this work, we focus on prompt photon production in proton-proton and lepton-proton scattering. Transverse single spin asymmetry (TSSA) is defined as

$$
A_{N}=\frac{d \sigma^{\uparrow}-d \sigma^{\downarrow}}{d \sigma^{\uparrow}+d \sigma^{\downarrow}}
$$

where $d \sigma^{\uparrow(\downarrow)}$ is the invariant differential cross-section with the spin of the transversely polarized proton being aligned in the $\uparrow(\downarrow)$ direction with respect to the production plane. We make predictions for asymmetry using first the generalized paron model (GPM) [1] and then using color gauge invariant generalized parton model (CGI-GPM) [2, 3]. Assumptions implicit in GPM are that (i) transverse momentum dependent (TMD) factorization holds for single hard scale process also and (ii) TMDs are process independent. In GPM, the numerator of asymmetry in Eq.1.1, for the direct process $p^{\uparrow} p \rightarrow \gamma+X$, is

$$
\begin{aligned}
d \sigma^{\uparrow}-d \sigma^{\downarrow} & =\frac{\pi \alpha_{s} \alpha_{e m}}{\hat{s}^{2}} \sum_{a, b=g, q, \bar{q}} \int d x_{a} d^{2} \mathbf{k}_{\perp a} d x_{b} d^{2} \mathbf{k}_{\perp b} \Delta^{N} f_{a / p^{\uparrow}}\left(x_{a}, \mathbf{k}_{\perp a}\right) \hat{f}_{b / p}\left(x_{b}, k_{\perp b}\right) \\
& \times \frac{\hat{s}}{x_{a} x_{b} s} H_{a b \rightarrow \gamma d}^{U} \frac{\hat{s}}{\pi} \delta(\hat{s}+\hat{t}+\hat{u})
\end{aligned}
$$

where $H_{a b \rightarrow \gamma d}^{U}$ is the hard part, $\hat{f}_{b / p}$ is a transverse momentum dependent parton distibution function (TMD-PDF) describing distribution of unpolarised parton b inside unpolarised proton, and $\Delta^{N} f_{a / p^{\uparrow}}$ is the Sivers function given by,

$$
\begin{aligned}
\Delta^{N} f_{a / p^{\uparrow}}\left(x_{a}, \mathbf{k}_{\perp a}\right) & =\hat{f}_{a / p^{\uparrow}}\left(x_{a}, \mathbf{k}_{\perp a}\right)-\hat{f}_{a / p^{\downarrow}}\left(x_{a}, \mathbf{k}_{\perp a}\right) \\
& =\Delta^{N} f_{a / p^{\uparrow}}\left(x_{a}, k_{\perp a}\right) \cos \phi_{a}=-2 \frac{k_{\perp a}}{M_{p}} f_{1 T}^{\perp a}\left(x_{a}, k_{\perp a}\right) \cos \phi_{a}
\end{aligned}
$$

In the above equation, $\hat{f}_{a / p^{\uparrow(\downarrow)}}$ is TMD-PDF describing distribution of unpolarised parton inside transeversely upward(downward) proton.

Colour-Gauge Invariant Generalized Parton Model (CGI-GPM) takes into account effect of initial-state interactions (ISI) and final-state interactions (FSI) between the struck parton and spectators from the polarized proton thus making TMD densities process dependent. In this model, the process dependence of the Sivers function is taken into account by treating the initial state/final state interactions using a single eikonal-gluon exchange approximation, i.e., one has to use modified hard parts with the following replacement for QSFs:

$$
f_{1 T}^{\perp} H_{q b \rightarrow c d}^{U} \rightarrow f_{1 T}^{\perp} H_{q b \rightarrow c d}^{m o d}=\frac{C_{I}+C_{F c}}{C_{U}} f_{1 T}^{\perp} H_{q b \rightarrow c d}^{U}
$$

where $C_{U}$ is the color factor corresponding to unpolarized cross section, $C_{I}$ and $C_{F c}$ are the colour factors for the diagrams involving ISI and FSI respectively and $f_{1 T}$ is the Sivers function probed in SIDIS.

In case of gluon Sivers function(GSF), the process-dependent GSF is a linear combination of two independent universal gluon distributions $f_{1 T}^{\perp g(f)}$ and $f_{1 T}^{\perp g(d)}$, which correspond to the two 
different ways in which color can be neutralized. Therefore for the case of the GSF the following substitution is required:

$$
\begin{aligned}
f_{1 T}^{\perp g} H_{g b \rightarrow c d}^{U} \rightarrow f_{1 T}^{\perp g} H_{g b \rightarrow c d}^{m o d} & =\frac{C_{I}^{f}+C_{F c}^{f}}{C_{U}} f_{1 T}^{\perp g(f)} H_{g b \rightarrow c d}^{U}+\frac{C_{I}^{d}+C_{F c}^{d}}{C_{U}} f_{1 T}^{\perp g(d)} H_{g b \rightarrow c d}^{U} \\
& =f_{1 T}^{\perp} H_{g b \rightarrow c d}^{(f)}+f_{1 T}^{\perp} H_{g b \rightarrow c d}^{(d)}
\end{aligned}
$$

Feynman rules for calculateing modified Hard parts in CGI-GPM formalism are given in Ref.[4] and the modified Hard parts for the processes considered here can be found in Refs.[2, 4, 5].

We adopt the functional form of the Sivers functions used in of Ref.[6]:

$$
\Delta^{N} f_{i / P^{\uparrow}}\left(x, k_{\perp} ; Q\right)=2 \mathscr{N}_{i}(x) f_{i / p}(x, Q) \frac{\sqrt{2 e}}{\pi} \sqrt{\frac{1-\rho}{\rho}} k_{\perp} \frac{e^{-k_{\perp}^{2} / \rho\left\langle k_{\perp}^{2}\right\rangle}}{\left\langle k_{\perp}^{2}\right\rangle^{3 / 2}}
$$

with $0<\rho<1$. Here $\mathscr{N}_{i}(x)$ parametrizes the $x$-dependence of the Sivers function. For the Sivers function to satisfy the positivity bound,

$$
\frac{\left|\Delta^{N} f_{i / p^{\uparrow}}\left(x, \mathbf{k}_{\perp}\right)\right|}{2 f_{i / p}\left(x, \mathbf{k}_{\perp}\right)} \leq 1 \quad \forall x, \mathbf{k}_{\perp},
$$

it is necessary to have $\left|\mathscr{N}_{i}(x)\right| \leq 1$.

In order to study the efficacy of the probe and the relative sizes of the contributions from the quarks and gluons, we have used both quark and gluon Sivers functions with the positivity bound saturated, viz. $\mathscr{N}_{i}(x)=1$, and also set $\rho=2 / 3$.

\section{Prompt Photons at RHIC}

In this section, we present the results of TSSA in prompt photon production in $p^{\uparrow} p$ scattering. Prompt photons in proton-proton collisions have two contributions: (a)Direct contribution, where photons are directly produced at the hard scattering level through QCD Compton process, $g q \rightarrow$ $\gamma q$ and quark-antiquark annihilation process $q \bar{q} \rightarrow \gamma g$, which are of the order $\mathscr{O}\left(\alpha_{e m} \alpha_{s}\right)$ and (b) Fragmentation contributions, where photons are produced at leading order through the standard 2to-2 QCD parton scattering processes with the final-state parton fragmenting into a photon $(q, g \rightarrow$ $\gamma+X)$. Although partonic processes involved in fragmentation contribution are of the order $\mathscr{O}\left(\alpha_{s}^{2}\right)$, the parton-to-photon FFs have a leading order behavior propotional to $\alpha_{e m} / \alpha_{s}$, which makes the fragmentation photons effectively at the same leading order $\mathscr{O}\left(\alpha_{e m} \alpha_{s}\right)$ as the production of direct photons. We show in Fig. 1 the unpolarised Lorentz-invariant cross-section for prompt photon production at RHIC energy $\sqrt{s}=200 \mathrm{GeV}$ as a function of $x_{F}$ at $P_{T}=5 \mathrm{GeV}$ in the left panel and as a function of $P_{T}$ at rapidity $\eta=-2$ in the right panel. We find that both the direct and the fragmentation contributions are of the same order of magnitude and that the gluon initiated processes have the dominant contribution to the cross-section in the region considered here.

In Fig.2, we show the prediction of TSSA in prompt photon production at RHIC using GPM in left panel and using CGI-GPM in right panel. We find that the use of saturated gluon Sivers function can lead to an asymmetry in direct photons of up to $10 \%$ in GPM. We find that inclusion of fragmentation photons can dilute the asymmetries. We find that both the f-type and d-type GSFs 

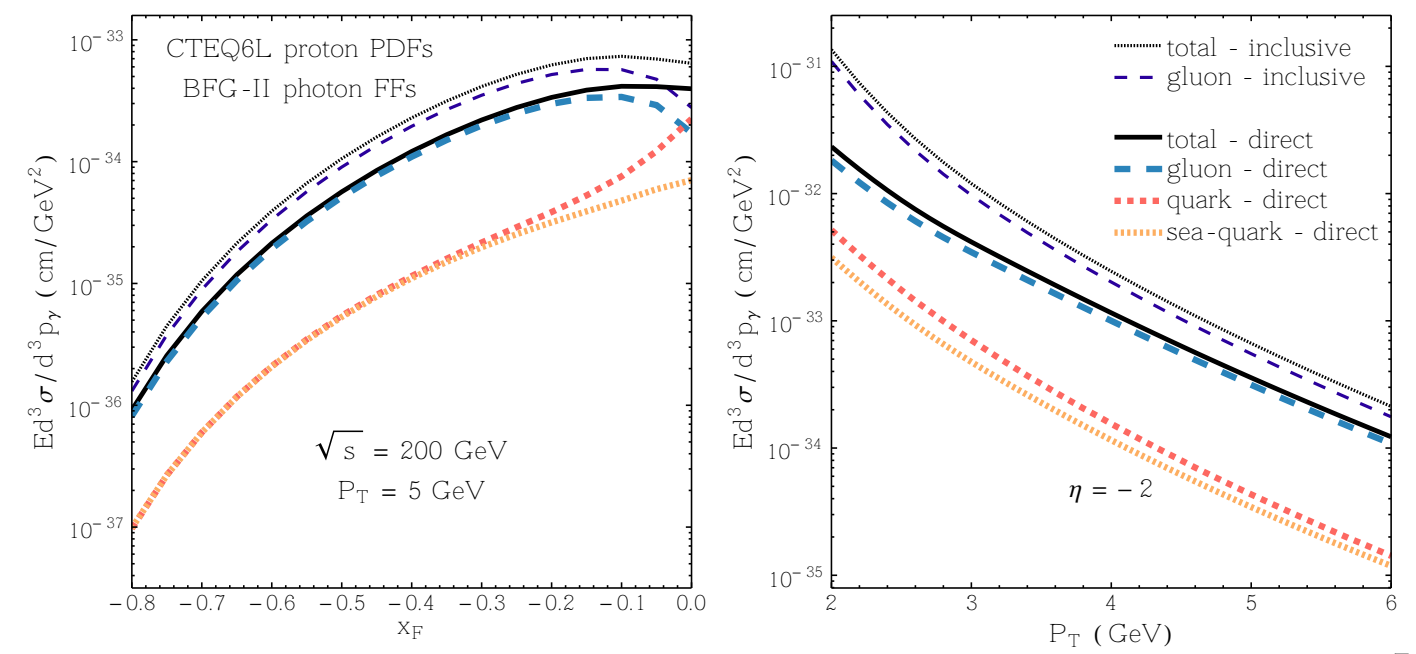

Figure 1: Unpolarised Lorentz-invariant cross-section for prompt photon production at RHIC $(\sqrt{s}=200$ $\mathrm{GeV}$ ) as a function of $x_{F}$ (at $P_{T}=5 \mathrm{GeV}$, left panel) and $P_{T}$ (at rapidity $\eta=-2$, right panel). Figure from Ref. [5]
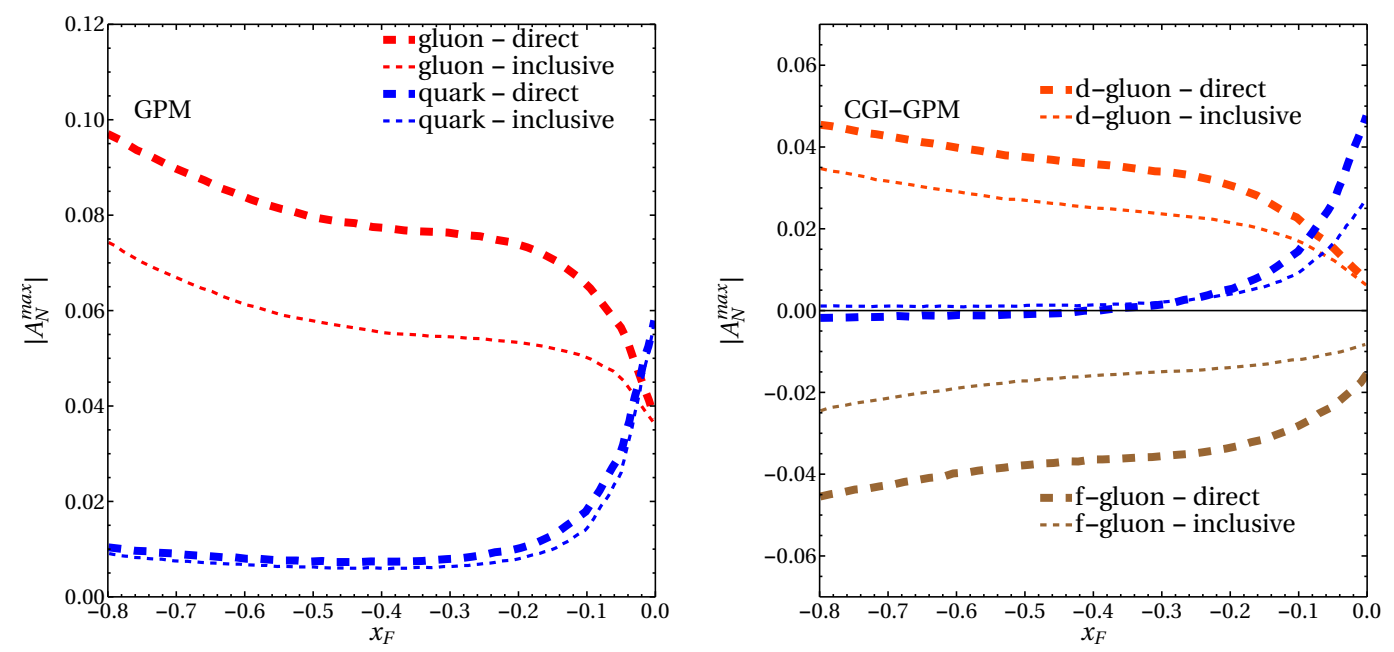

Figure 2: SSA in direct photon production with saturated quark and gluon Sivers functions at RHIC $(\sqrt{s}=$ $200 \mathrm{GeV}$ ) as a function of $x_{F}$ (using the GPM framework, left panel) and $P_{T}$ (using the CGI-GPM framework, right panel). Figures from Ref. [5]

in CGI-GPM contribute to TSSA up to 5\%. Also, the gluon contribution to asymmetry is dominant relative to quark contribution in the region considered here. Thus, any non zero measurement of TSSA in this region would imply a non-zero gluon Sivers function.

\section{Prompt Photons at EIC}

In this section, we present a preliminary study of asymmetry in $p^{\uparrow} e$ scattering. In this case, photons can be produced through direct subprocess $\gamma q \rightarrow \gamma q$ or through resolved processes in which partons originating from initial photon take part in the hard scattering where the lowest order processes are $q \bar{q} \rightarrow \gamma g$ and $q g \rightarrow \gamma q$. In the current analysis, we have not considered fragmentation contribution, which can be excluded by applying isolation cuts in the experiment.

In Fig.3, we present $x_{F}$ distribution of the unpolarized cross-section for production of prompt 
photons at EIC energy, $\sqrt{s}=140 \mathrm{GeV}$ at fixed $p_{T}=3 \mathrm{GeV}$ in the left panel and saturated asymmetry in the right panel. We see that in the region considered here, the cross-sction for direct subprocess is greater than that of resolved subprocesses. From the right panel of Fig.3, we see that the asymmetry in generelized parton model gets a dominant contibution i.e. up to $13 \%$ from quark Sivers function while gluon Sivers function contributes up to $3 \%$ to $4 \%$ to the asymmetry. When we calculate asymmetry using CGI-CPM aproach, the situation changes drastically. Here, we get negligible asymmetry due to quark Sivers function because of the fact that direct subprocess $q \bar{q} \rightarrow \gamma g$, which has a dominant contribution to the cross section in the region considered here, does not contribute to the numerator of the asymmetry in CGI-GPM due to the absence of ISI and FSI. However, this direct subprocess does contribute to the denominator of the asymmetry which is twice the unpolarized cross section. Another interesting feature of this process is that the contribution from the d-type GSF to the asymmetry is zero because the modified hard parts have opposite signs for quarks and antiquarks $H_{g q \rightarrow \gamma q}^{(d)}=-H_{g \bar{q} \rightarrow \gamma \bar{q}}^{(d)}$ and the distributions for quarks and antiquaks are same for the photon. Hence, measuring the asymmetry in prompt photon production at EIC would shed some light on the as yet poorly unknown gluon Sivers function. The detailed analysis of this process can be found in [7].
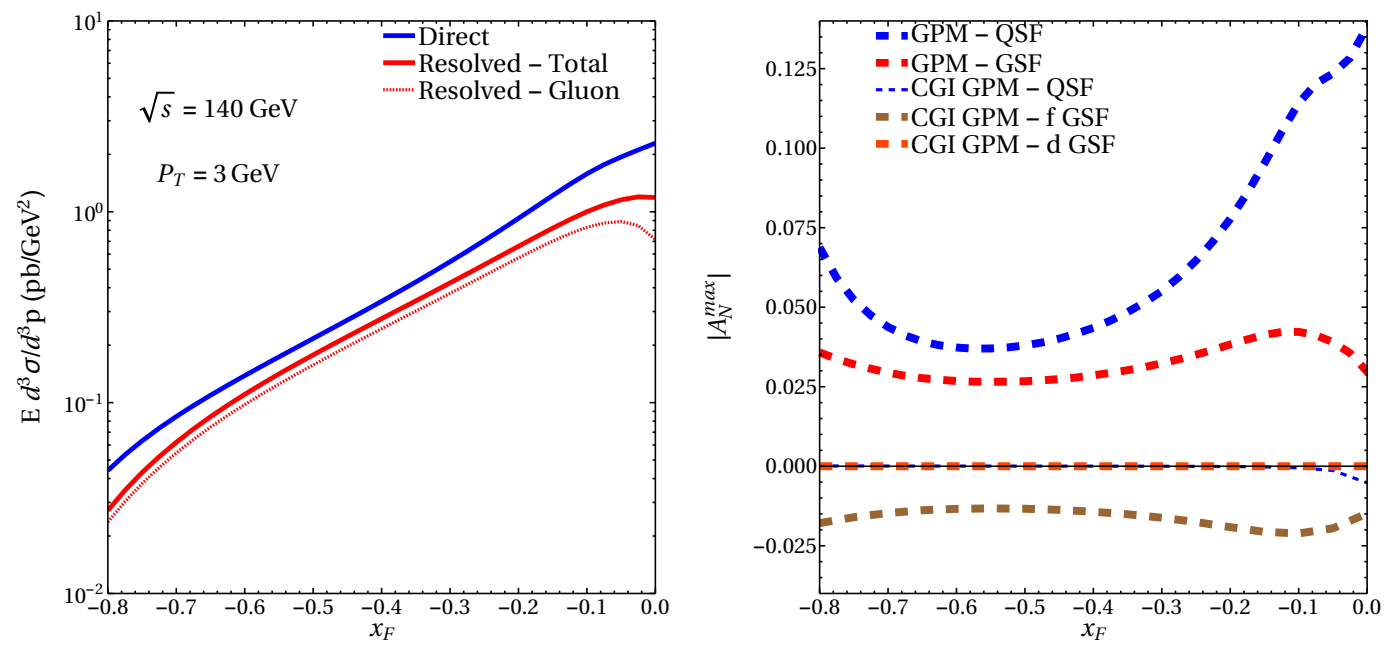

Figure 3: Unpolarised Lorentz-invariant cross-section for prompt photon production at EIC $(\sqrt{s}=140$ $\mathrm{GeV}$ ) as a function of $x_{F}$ at $P_{T}=3 \mathrm{GeV}$, left panel and estimates of $\left|A_{N}^{\max }\right|$ using saturated quark and gluon Sivers function at right panel. Plots from [7].

\section{Conclusion}

Our study suggests that measurement of asymmetry in prompt photon production both at RHIC and EIC would be useful for the extraction of gluon Sivers function. We find that in case of RHIC, saturated gluon Sivers function gives asymmetry up to $10 \%$ in generalized parton model, while using CGI-GPM framework, we find that both f-type and d-type GSFs contribute and lead to 5\% asymmetry. Further, we also studied the asymmetry in prompt photon production at EIC. We find that predictions of asymmetries calculated using GPM and CGI-GPM frameworks are completely different. Whereas we find asymmetry up to $10 \%$ from QSF and up to $2 \%$ from GSF in GPM, in case of CGI-GPM, we find asymmetry up to $1 \%$ from f-type GSF and negligible asymmetry from the QSFs because of the fact that the direct subprocess $\gamma q \rightarrow \gamma q$ - which has a dominant contri- 
bution to the cross-section in the region considered here - does not contribute to the asymmetry due to the absence of ISI and FSI. We find that d-type GSF does not contribute to the asymmetry in this process. This fact would be useful to constrain f-type GSF and to distinguish between the GPM and CGI-GPM models.

\section{References}

[1] U. D'Alesio and F. Murgia, Azimuthal and single spin asymmetries in hard scattering processes, Progress in Particle and Nuclear Physics 61 (2008) 394 [arXiv:0712.4328].

[2] L. Gamberg and Z.-B. Kang, Process dependent sivers function and implication for single spin asymmetry in inclusive hadron production, Physics Letters B 696 (2011) 109 [arXiv:1009.1936].

[3] U. D'Alesio, L. Gamberg, Z.-B. Kang, F. Murgia and C. Pisano, Testing the process dependence of the sivers function via hadron distributions inside a jet, Physics Letters B 704 (2011) 637 [arXiv:1108.0827].

[4] U. D' Alesio, F. Murgia, C. Pisano and P. Taels, Probing the gluon sivers function in $p^{\uparrow} p \rightarrow j / \psi x$ and $p^{\uparrow} p \rightarrow d x$, Phys. Rev. D 96 (2017) 036011 [arXiv:1705.04169].

[5] R. M. Godbole, A. Kaushik, A. Misra and S. Padval, Probing the gluon sivers function through direct photon production at rhic, Phys. Rev. D 99 (2019) 014003

[arXiv:1810.07113].

[6] U. D'Alesio, F. Murgia and C. Pisano, Towards a first estimate of the gluon sivers function from andata in pp collisions at rhic, Journal of High Energy Physics 2015 (2015) 119 [arXiv:1506.03078].

[7] S. Padval, R. M. Godbole, A. Kaushik, A. Misra and V. S. Rawoot, Gluon sivers function and transverse single spin asymmetries in $e+p^{\uparrow} \rightarrow \gamma+x$, arXiv:2002.01282. 